\title{
SETTINGS ADAPTATION SYSTEM FOR CURRENT PROTECTION RELAY
}

\author{
Ualikhan Iskakov, Josif Breido
}

Bulvar Mira 56, main bld. 140, 100027 Karaganda, Kazakhstan

\begin{abstract}
This paper proposes the new approaches and technology of development an adaptive to voltage oscillation overcurrent protection. The model of settings adaptation system was developed using Matlab SIMULINK, this model allows changing settings of relay protection during voltage oscillation and allows preventing non-selective (tripping) operation. The generalized model of overcurrent protection and the model of isolated neutral system were developed. The model of isolated neutral system allows simulating the model of overcurrent protection in operating and emergency conditions of power systems and allows making assessment of adaptive relay protections.
\end{abstract}

Keyword: Overcurrent protection; Settings; Adaptation; Voltage oscillation; Simulation modelling
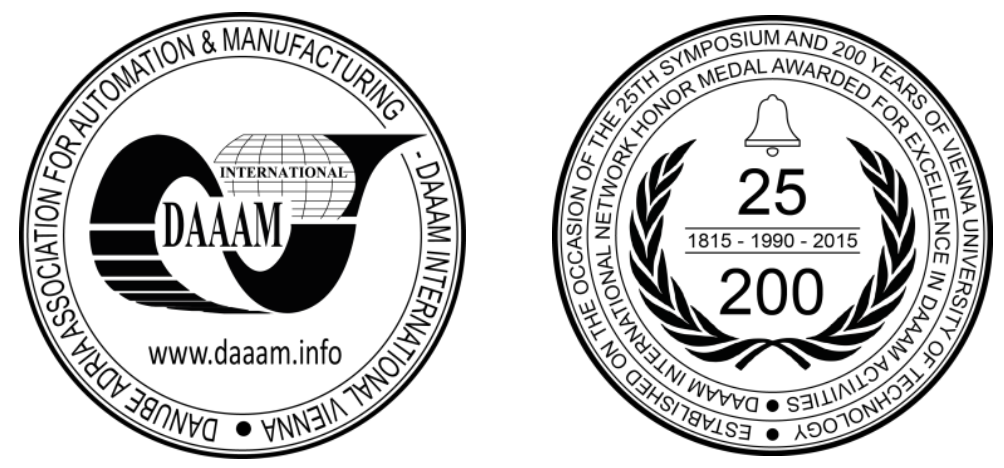

This Publication has to be referred as: Iskakov, U[alikhan] \& Breido, J[osif] (2016). Settings Adaptation System for Current Protection Relay, Proceedings of the 26th DAAAM International Symposium, pp.0371-0377, B. Katalinic (Ed.), Published by DAAAM International, ISBN 978-3-902734-07-5, ISSN 1726-9679, Vienna, Austria DOI:10.2507/26th.daaam.proceedings.050 


\section{Introduction}

Microprocessor based protection relays are utilized for safe reliable and efficient operation of power systems, and also to prevent the detrimental effects of faults. The current tasks solved by overcurrent protection have high responsibility. Therefore it requires from protection relays reliable and stable operation, measurement accuracy of power system parameters and characteristics stability. General shortcoming of existing protection relays is unadaptable settings during changing of operation conditions and changing of electric network parameters. Wrong settings can lead to unselective or false operation of protection relay [1]. Protection relays should possess adaptive properties which change according to network's characteristics [2].

The control algorithm and model of settings adaptation system were developed. The model of adaptation system registers changing of electric network parameters and makes correction of settings which was previously calculated for nominal conditions. The settings correction is calculated according to determined dependence of settings on changing electric network parameters, separately for each type of protection (instantaneous overcurrent protection, definite time overcurrent protection, earth fault protection).

\section{Functional chart of adaptive current protections system}

The modern protection relays operate using settings calculated for nominal conditions. New developments in microprocessor based protection relays allow to integrate protection systems with data acquisition and processing to adapt settings according to changing of operation conditions and changing of electric network parameters in real time mode [3].

The settings of modern protection relays should be adaptive to changing of electric network parameters, as follows [4]:

1. Voltage of electric network;

2. Rated current;

3. Length of controllable line;

4. Resistivity of power line conductors;

5. Type of electrical load.

To control aforementioned parameters it is reasonable to use information from measurement transformers (current, voltage, zero sequence current) used as basic elements of protection relay's measuring unit [5]. For the adaptation system as input signal can be used data from protection relay's measuring unit. The data of measuring unit is taken from sensors of electric network parameters. The output signal of adaptation system can be transferred to protection relays using system interfaces [6] widely used in modern microprocessor based protection relay units.

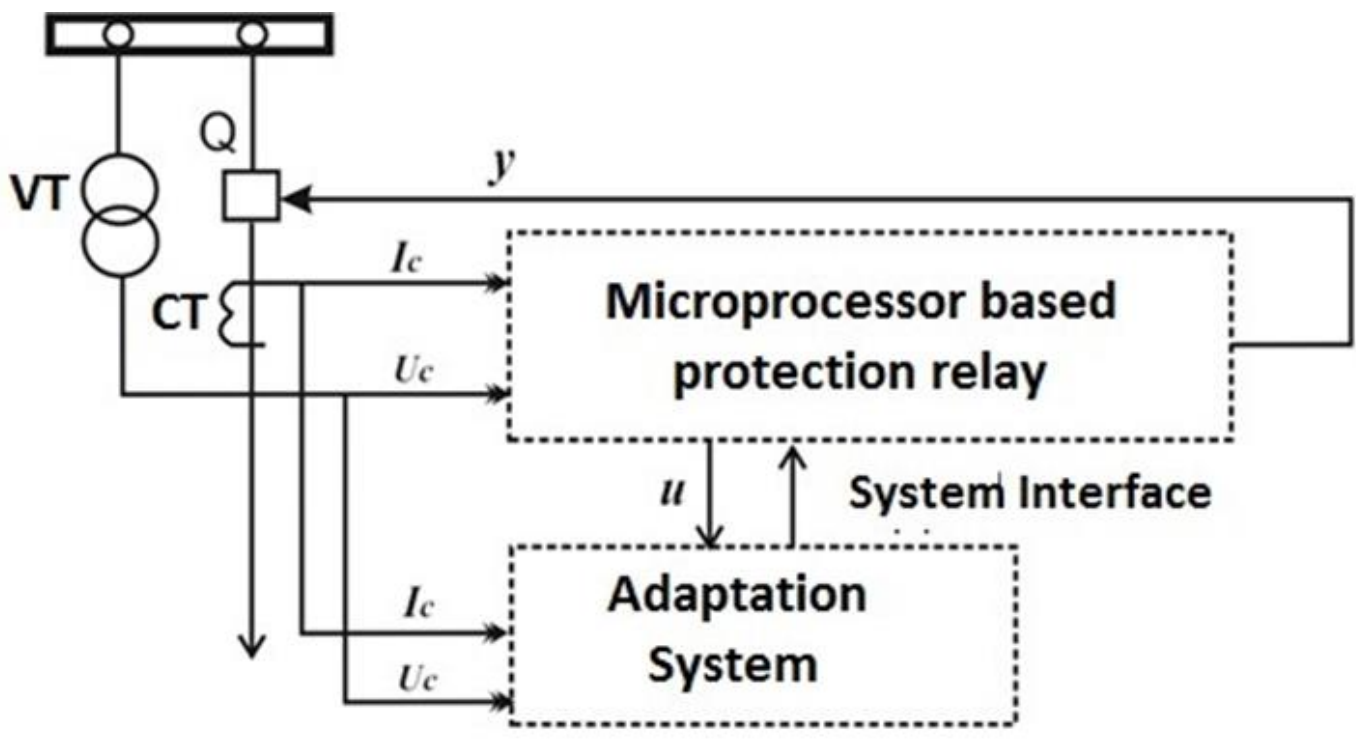

Fig. 1. Functional chart of adaptation system

The functional diagram, explaining operation principle of adaptation system, shown on Fig.1. The adaptation system receives signals from current transformers and voltage transformers, then registers changing of electric network parameters and calculates correction signal for settings, this signal is passed to protection relays via system interfaces. 


\section{Determination of functional criteria and development of adaptation model}

Developed adaptation model intend to instantaneous overcurrent protection, definite time overcurrent protection and earth fault protection. Electric network voltage was chosen as the controllable parameter for settings adaptation.

The pick-up current of definite time overcurrent protection is calculated as follows eq.(1):

$$
I_{p i c k-u p}=\frac{C_{r e l} \cdot C_{i . c . r .}}{C_{d . r .}} \cdot I_{r . c .}
$$

Where:

$C_{r e l}-$ is the reliability index of relay protection;

$C_{\text {i.c. } . \text {. }}$ - is the inrush current ratio;

$C_{d . r .}-$ is the drop-out ratio of relay protection;

$I_{\text {.r.c. }}-$ is the rated current of electric network.

The product of rated current and inrush current ratio gives maximal rated current $I_{m . r . c .}$ eq.(2):

$$
I_{\text {m.r.c. }}=C_{i . c . r .} \cdot I_{r . c .}=\frac{U}{\sqrt{3} \sum x}
$$

Where:

$U-$ is the linear voltage of electric network;

$\sum \mathrm{x}$ - is the total inrush inductive reactance of electric motor and feeding power supply and electric line.

Starting reactance of electric motor is calculated as follows eq.(3) [7]:

$x_{\text {s.r. }}=\frac{U}{\sqrt{3} I_{\text {i.c.e. }}}=\frac{U}{\sqrt{3} I_{r . . c .} \cdot C_{i . c . r .}}$

Aforementioned equations show direct proportionality of maximal rated current from network voltage.

Determining equation of pick-up current for definite time overcurrent protection will look as follows eq.(4):

$$
I_{\text {pick-up }}=\frac{U \cdot C_{r e l}}{C_{d . r .} \sqrt{3} \sum x}
$$

During voltage oscillation maximal rated current is changed and settings of definite time overcurrent protection should be changed also.

The pick-up current of instantaneous overcurrent protection is calculated as follows eq.(5):

$$
I_{p i c k-u p}=\frac{U \cdot C_{o f f s e t}}{\sqrt{3} \sum x}
$$

Where:

$C_{\text {offfset }}$ - is the offset coefficient of protection relay which is equal to $1,1-1,2$ for microprocessor based protection relay. During voltage oscillation short-circuit current is changed and settings of instantaneous overcurrent protection should be changed also.

The pick-up current of earth fault protection in isolated neutral system is equal to a triple capacitive current of network eq.(6):

$$
I_{\text {pick-up }}=-3 \underline{E}_{A} / X_{C}=-j 3 U_{A} / X_{C}
$$


Where:

$E_{A}-$ is the electromotive force of phase;

$X_{C}-$ is the capacitive reactance of phase;

$U_{A}-$ is the phase voltage.

The capacitive current of network is directly proportional to voltage of electric network. During voltage oscillation capacitive current of network is changed and settings of earth fault protection should be changed also.The settings of current protections (instantaneous, definite time and earth fault) have common (direct proportional) dependability to voltage of electric network. Therefore universal settings adaptation system was developed.

\section{Development of generalized simulation model of overcurrent protection}

The analysis of the operation principles and functional structures of microprocessor based protections [8, 9, 10, 11] like instantaneous overcurrent protection, definite time overcurrent protection and earth fault protection revealed that all of them could be presented in one generalized model. The generalized simulation model of overcurrent protection, implemented using SimPowerSystems (MATLAB/Simulink) [12] interactive tool, showed on fig.2.

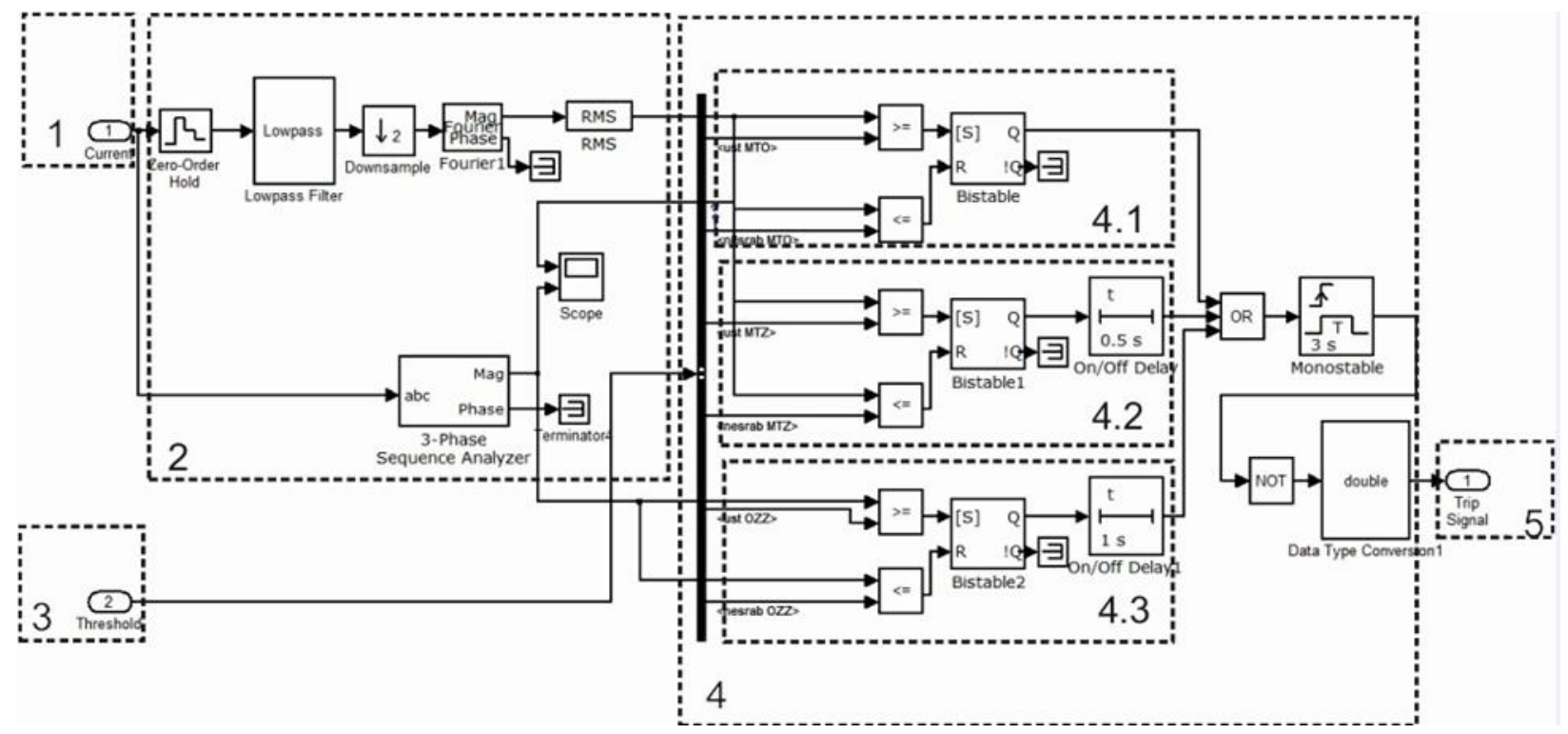

Fig. 2. Simulation model of overcurrent protection device: 1 - analog inputs of three-phase current; 2 - converter unit (CU); 3 - settings unit (Threshold); 4 - logic unit (LU) 4.1 instantaneous overcurrent protections LU, 4.2 - definite time overcurrent protection LU, 4.3 - earth fault protection LU; 5 - output signal to the switching equipment

To the inputs of model three-phase current value and zero sequence current value are coming up. The converter unit realizes analog-to-digital converting using Zero Order Hold block. To noise filtering Low pass Filter block is used. Down Sample block is used in order to reduce sampling rate, it is helps avoiding anti-aliasing effects. The Fourier block is used to extract periodic component of short circuit current. The 3-Phase Sequence Analyzer block is used to obtain zero-sequence-current. Protection algorithm is realized using comparison and Bistable trigger blocks, time delay is realized using On/Off Delay block. Obtained short circuit current and zero-sequence-current values are compared with threshold value, which are come from threshold settings block. The overcurrent device output produces control signal (Trip Signal) to switching device.

\section{Development of adaptation system for overcurrent protection}

For adaptation of current protections settings, settings calculated for nominal conditions should be multiplied to proportional coefficient which shows variation of voltage network. The model of settings adaptation system is shown on Fig. 3. 


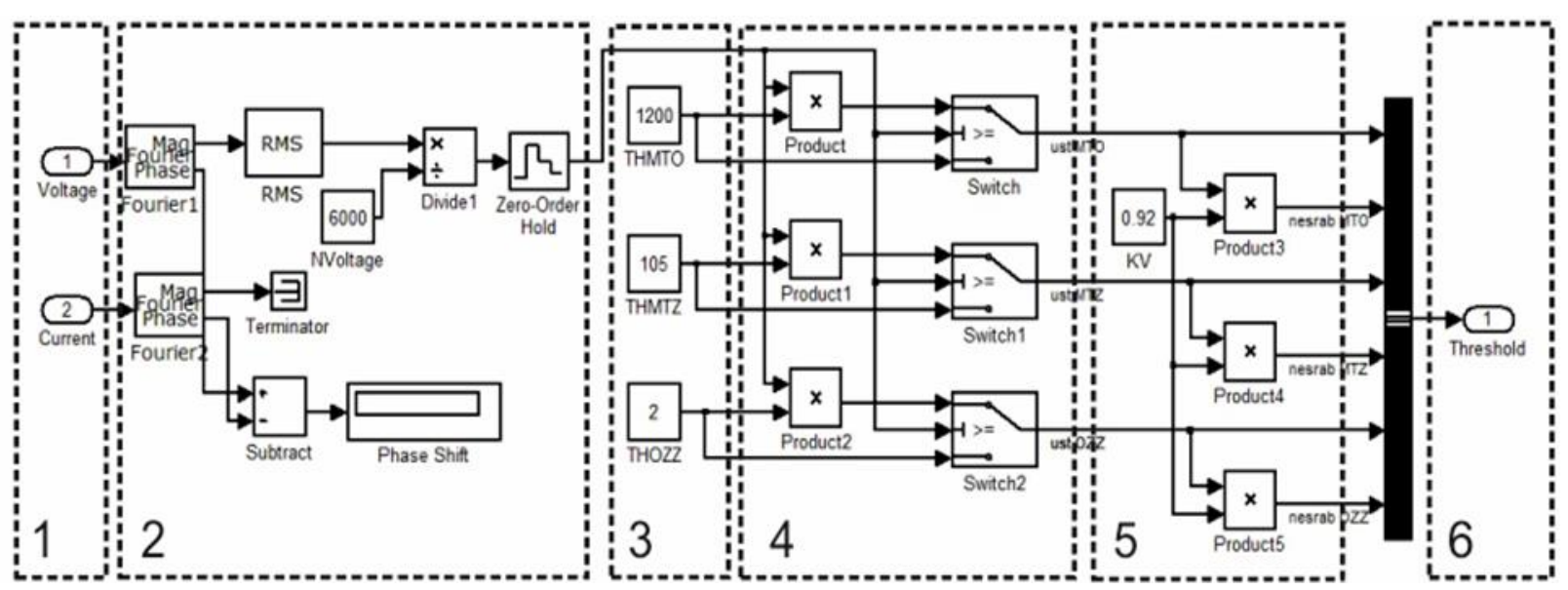

Fig. 3. The model of settings adaption system for current protections: 1 - input of analog current and analog voltage values: 2 - proportional coefficient calculation unit; 3 - calculated for rated operation settings adjuster; 4 - switching unit from calculated to nominal conditions settings to adaptive settings; 5 - drop-out ratio adjuster unit; 6 - settings transferring unit to protection relay

The adaptation system calculates ratio of effective voltage value to rated voltage value, which has been used to estimate settings for nominal conditions. Then, obtained proportional coefficient is multiplied to nominal conditions settings. Drop-out settings for relay protection are calculated using drop-out ratio.

\section{Operability analysis of settings adaptation system}

Simulation modelling was carried out to make operability assessment of settings adaptation system. The model of isolated neutral system (Fig.4) was developed to simulation modelling. This system was chosen due to its widespread in medium voltage networks and the superiority of isolated networks in terms of availability [13].

The model of isolated neutral system shown on Fig.4 consists of following major components:

- Three-phase Programmable Voltage Source with time-varying parameters $50 \mathrm{~Hz}, 6 \mathrm{kV}$ phase voltage;

- Distributed Parameters line transmission model with lumped losses 0,01273 Ohm/km, 0,9337 mH/km, 12,74 $\mu \mathrm{F}, 1 \mathrm{~km}$;

- Three-phase asynchronous machine $6 \mathrm{kV}, 50 \mathrm{~Hz}, 630 \mathrm{kVA}, 1725 \mathrm{rpm}$;

- Three-phase fault blocks, placed in the beginning and in the end of line, introduce single phase to ground, phase to phase and three phase faults;

- Model of settings adaptation system;

- The generalized simulation model of overcurrent protection.

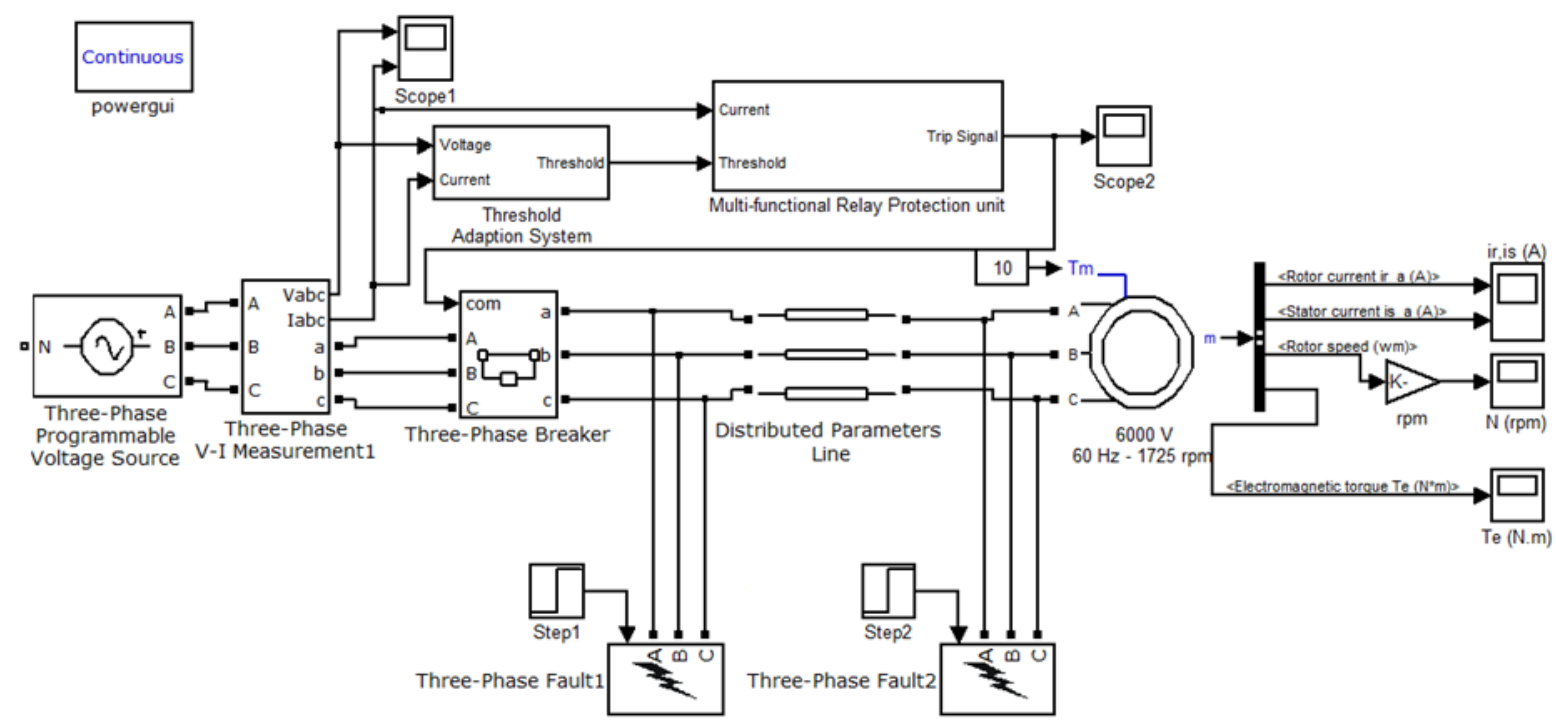

Fig. 4.The model of isolated neutral system 
Modeling was carried out in two simulation conditions:

- Modelling of overcurrent protection operation in case of three-phase metal short circuit without settings adaptation system;

- Modelling of overcurrent protection operation in case of three-phase metal short circuit with active settings adaptation system.

On Fig. 5. are presented results of modeling in first simulation condition. There are graphs of instantaneous overcurrent protection settings and phase voltage of electric network during voltage increasing to $15 \%$ from rated value. Phase voltage stepwise changing to $15 \%$ is shown on the top graph. Settings of instantaneous overcurrent protection are shown on lower graph.

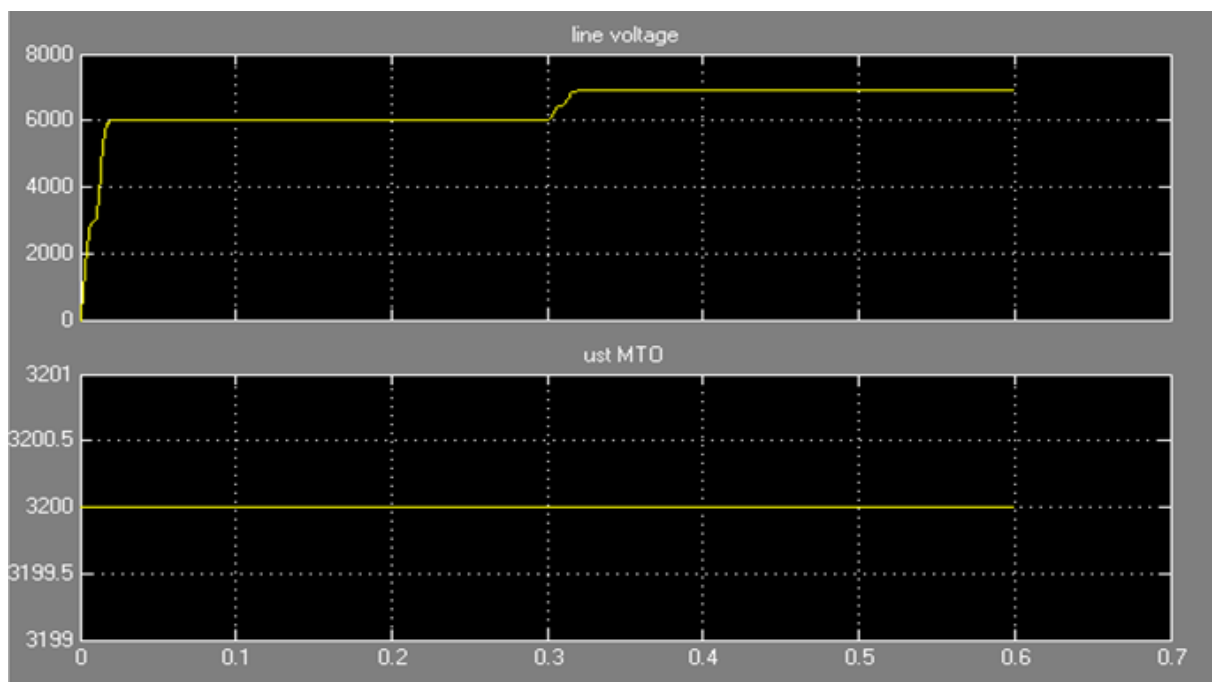

Fig. 5. Graph of settings of instantaneous overcurrent protection and phase voltage of electric network during voltage increasing to $15 \%$ from rated value

In this case short-circuit current value is changed direct proportional to voltage changing, nevertheless settings of relay protection remained invariable that leads to non-selective operation of protection relay during faults on upstream protected line.

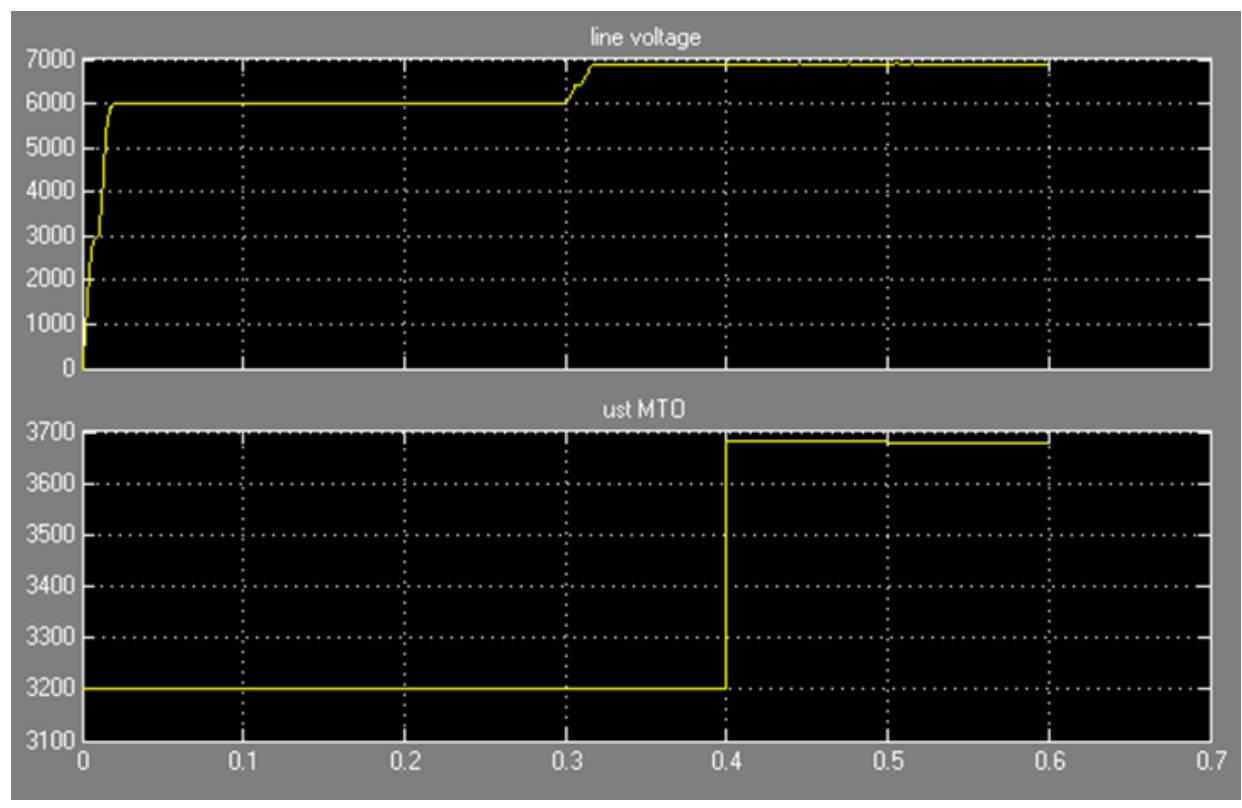

Fig. 6. Graph of settings of instantaneous overcurrent protection and phase voltage of electric network during voltage increasing to $15 \%$ from rated value with active settings adaptation system 
On Fig.6 are presented results of modeling in second simulation condition. There are graphs of instantaneous overcurrent protection settings and phase voltage of electric network during voltage increasing to $15 \%$ from rated value with active settings adaptation system. Voltage value is come to input of settings adaptation system. In this case settings of relay protection increase from 3200 A (ampere) to 3680 A. New settings are calculated using proportional coefficient which shows varying of network voltage. New settings ensure selective operation of relay protection.

As shown on fig. 6 adaptation of protection relay settings is ensued with $0.1 \mathrm{sec}$ time delay, specified by operation of Zero Order Hold block. Zero Order Hold block makes time sampling of input signal, thereby adaptation of protection relay settings is prevented during short-time voltage oscillations. Adaption system changes settings of relay protection during voltage oscillation and prevent non-selective (tripping) operation of relay protection.

\section{Conclusion}

Developed isolated neutral system model allows simulating operation and emergency conditions of developed generalized model of overcurrent protection, and also gives possibility to control parameters, which are influence to protection device tripping. The simulation model of overcurrent protection allows validating calculated threshold values and settings, and also allows detecting power system conditions which lead to malfunctioning of protection devices.

Developed model of settings adaptation system allows changing settings of relay protection during voltage oscillation and preventing non-selective (tripping) operation of relay protection. Adaptation of relay protection settings ensures enhancement of reliability and efficiency of protection relay devices [14].

\section{References}

[1] V. Calderaro, V. Galdi, A. Piccolo, P. Siano, Adaptive relays for overhead line protection, Electric Power Systems Research 77 (2007) 1552-1559, https://www.sciencedirect.com, 2006.

[2] J.D. Codling, S.A. House, J.H. Joice, K.M. Labhart, J.R. Richards, J.E. Tenbusch, Adaptive relaying. A new direction in power system protection, Potentials IEE, Volume 15 issue 1,ISSN: 0278-6648, 1996.

[3] Byung Chul Shung, Soo Hyoung Lee, Jung-Wook Park, A.P.S. Meliopoulos, Adaptive Protection Algorithm for Overcurrent Relay in Distribution System with DG, ISSN(Print) 1975-0102, J Electr Eng Technol V ol. 8, No. 5: 1002-1011, 2013.

[4] J. Breido, U. Iskakov, The adaptive tools of power system protection and electric equipment protection, Science and innovative developments - To the North, Mirnyi, 2014.

[5] C. Russell Mason, The Art \& Science of protective relaying, Published by Wiley, ISBN 10: 0471575526/ ISBN 13: $9780471575528,1956$.

[6] R. Jafari, M.S. Naderi , G.B. Gharehpetain, An Adaptive Protection Scheme based on Fault Location for Smart Micro-Grids, International Conference on Renewable Energies and Power Quality (ICREPQ'14) Cordoba (Spain), ISSN 2172-038 X, No.12, 2014.

[7] B. Metz-Noblat, F. Dumas, C. Poulain, Calculation of short-circuit currents, Cahier Tecnique Schneider Electric N $158,2005$.

[8] Operating instructions. Sepam + 1000 series 40. Protection functions 3/1. MerlinGerin Schneider Electric, pp 196197

[9] Operating instructions. Digital overcurrent protection relay with time delay and with thermal overload protection and autoreclosing SIPROTEC 7SJ602 v 3.1. Siemens AG 1999, pp 206-207 (2001)

[10] User manual (model R). MICOM P111 Universal overcurrent protection relay v 7B, pp 42-43

[11] Operating instructions. Digital protection block BMRZ-100, DIVG 648228.024UM, Mechanotronika, pp 35-36

[12] Christos A. Apostopopulos, George N. Korres, Real-time implementation of digital relay models using MATLAB/SIMULINK and RTDS, European Transactions on Electrical Power, https://www.interscience.wiley.com, 2008.

[13] S. Ravlić, A. Marušić, Simulation Models for Various Neutral Earthing Methods in Medium Voltage Systems, Procedia Engineering, Volume 100, 2015, pp 1182 - 1191.

[14] U. Iskakov, J. Breido, Development of control algorithm for adaptive leakage current protection devices' using Fuzzy logic, Procedia Engineering, Volume 100, 2015, pp 666-671. 